\title{
Difference Between Bone Healing Process with The Use Of Demineralized Freeze Dried Bone Xenograft and Bovine Bone Hydroxyapatite Xenograft Materials Zefry Zainal Abidin ${ }^{1 *}$, Coen Pramono², Achmad Harijadi², Aditiawarman ${ }^{3}$ \\ ${ }^{1}$ Faculty of Dentistry, Airlangga University \\ ${ }^{2}$ Department of Oral and Maxillofacial Surgery, Airlangga University \\ ${ }^{3}$ Fetomaternal Department of SMF Obstetrics and Gynecology, RSUD Dr. Soetomo Surabaya \\ *Corresponding author: smansasos@gmail.com
}

\begin{abstract}
Autogenous bone graft is the gold standard for bone defects treatment, however due to their limitation and the donor site morbidity may caused many surgeons use a xenograft type of bone grafting to cope the problem. Demineralized Freeze Dried Bone Xenograft(DFDBBX) which contains of growth factors, have a good biocompatibility. The aim of this study is observed the difference in bone healing processes between DFDBBX and Bovine Bone Hydroxyapatite Xenograft (BBHAX). Bicortical bone defects were created in the mandibular corpus of 30 New Zealand White Rabbits. The groups were divided into 3 groups which the first group were treated with DFDBBX into the hole and the negative control group was left perforated. The other group was treated with BBHAX. All group were evaluated after second and fourth weeks to count the ammount of osteoblast, osteoclast cells, Collagen-1 (Coll1) and alkalin phosphatase (ALP). The second week of observation showed a significant difference of mean 12,45, SD 2,97 ( $<<0,05)$ in osteoblast cells. In Coll-1 showed with mean 13,2 SD 2,68 ( $<<0,05)$. The result of ALP showed with mean 14,6 SD 2,70 ( $p<0,05)$. In the the fourth week observation showed increased of osteoclast cells with mean 7,043, SD 2,77 $(\mathrm{p}<0,05)$ and for Coll-1 with mean 17,6, SD $2,30(p<0,05)$. DFDBBX showed more effective in treating bone defects of mandible of new zealand white rabbits in second week of observation.
\end{abstract}

Keyword: Demineralized Freeze-Dried Bone Xenograft, Bovine Bone Hydroxyapatite Xenograft, Bone Healing, type I collagen and alkaline phospathase

\section{INTRODUCTION}

Maxillofacial bone defects caused by trauma or pathological conditions are health problems that need to be addressed because these conditions can have an impact on functional disorders (mastication, articulation), psychological and decreased quality of life of patients (Farzad \& Mohammadi, 2012). Defects in bone are distinguished based on the surface area of the defect, namely small or large (massive bone defects). One of the treatments for small bone defects is the provision of bone graft material (Ferdiansyah, 2007; Hernigou et al., 2005).

Osteogenesis is the formation and development of bone, and contains osteoblast 
cells which have the ability to produce bone matrix. Osteoinduction is the transformation of undifferentiated mesenchymal cells into osteoblasts or chondroblasts through growth factors that are only present in living bone, containing various cytokines such as transforming growth factor- $\beta$ (TGF- $\beta$ ), platelet derived growth factor (PDGF), insulin like growth factor (IGF), fibroblast growth factor (FGF), bone morphogenetic protein (BMP) which functions to attract, stimulate osteoprogenitor cells to proliferate and differentiate into osteoblasts which will then produce new bone.

Currently, there are 4 types of bone graft, namely autograft, allograft, xenograft, and alloplast (Hallman \& Thor, 2008). Autograft or autogenous bone graft is an ideal graft because it has osteogenic, osteoconductive, and osteoinductive properties needed for the bone regeneration process. Xenograft is an alternative

\section{METHOD}

This study is a true experimental study on experimental animals with a post test only control group design. The experimental unit in this study was a New Zealand White Rabbit rabbit that had been well conditioned before being used in the procedure. The experimental unit in this study was a New Zealand White Rabbit rabbit that had been well conditioned before being used in the procedure.

The sample size used in this study was obtained using the Frederer calculation formula, as follows: bone graft derived from the skeletal tissue of one species and implanted into another species in need. Generally, these grafts are derived from mammalian bone (Sandor, 2003).

Bovine bone hydroxyapatite xenograft is a bone graft derived from bovine bone that has been processed in a certain way so that it can be accepted by the body (Cohen et al., 1994; Hislop et al., 1993). This graft material is osteoconductive by inducing osteoblast activity in the formation of new bone (Buser, 2009). Demineralized freeze-dried bovine bone xenograft (DFDBBX) is an alternative bone graft material that still contains protein growth factors so that the bone matrix can be well regulated (Stephan et al., 1999). Biomarkers of bone formation are products resulting from osteoblast activity. Osteoblasts produce type 1 collagen and the matrix of other osteoid components. Biomarkers of bone formation can be measured in serum.

(n-1).(t-1) $\geq 15$

Description: $\mathrm{n}=$ number of replications; $\mathrm{t}=$ number of treatments so that if $(\mathrm{n}-1) \mathrm{x}(6-1)>$ 15 then $5 n-5>155 n>20$ then we get $n=4$

The formula for calculating the minimum number of samples mentioned above is used because there is no preliminary research. The number of treatments in this study was one treatment group and two control groups with a time of 2 observations so that the number of samples (n) was 4. Taking into account the possibility of excluded samples, the researchers used 5 samples for each treatment. The total 
number of samples is 30 samples.

\section{RESULTS}

This study was conducted to determine the potential for induction of osteogenesis in critical defects of the mandibular bone of rabbits after DFDBBX implantation with positive controls in the form of critical defects with BBHAX implantation and negative controls with critical defects without any implantation treatment with two different observation times.

The results of microscopic observations of osteoblast cells in the DFDBBX treatment, the BBHAX positive control group and the control group with $\mathrm{HE}$ staining during the second week of observation can be observed in the image below.

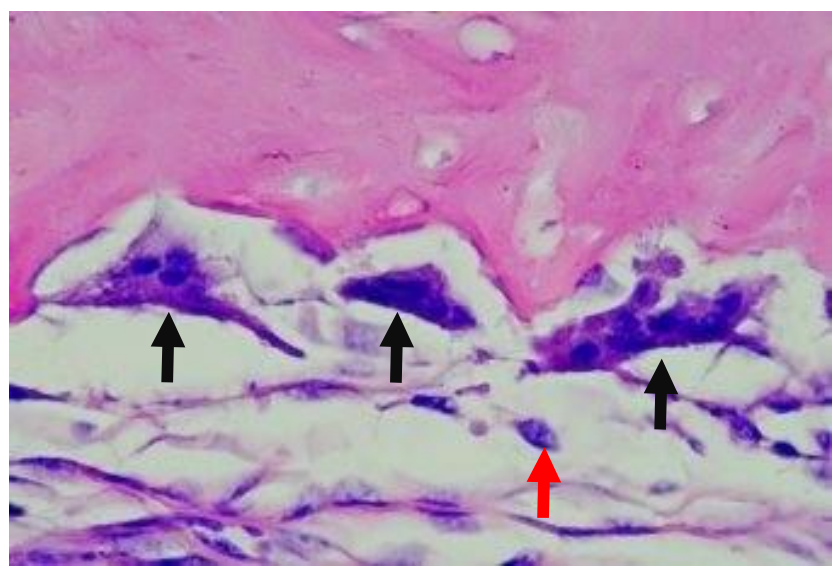

Figure 1. Microscopic images of osteoblasts and osteoclasts observed in week II in the negative control group (without graft).

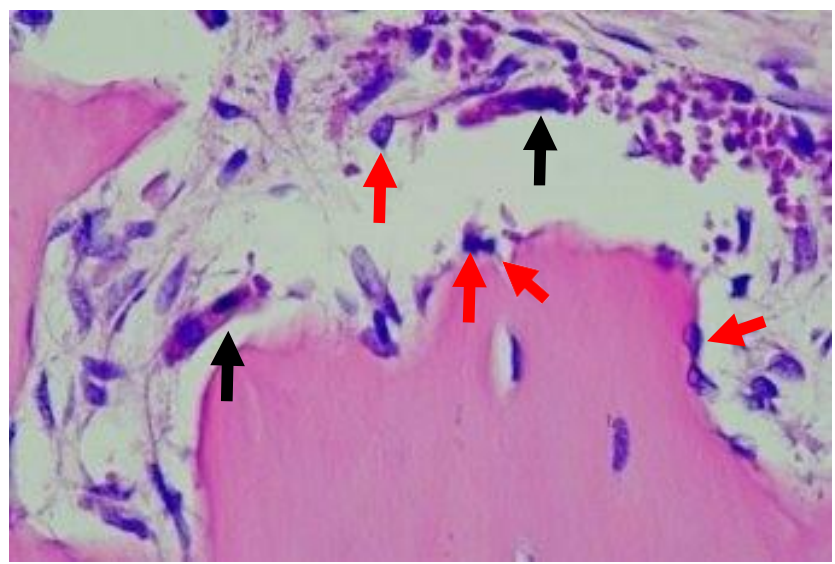

Figure 2. Microscopic images of osteoblasts and osteoclasts observed in week II in the positive control group (BBHAX) 


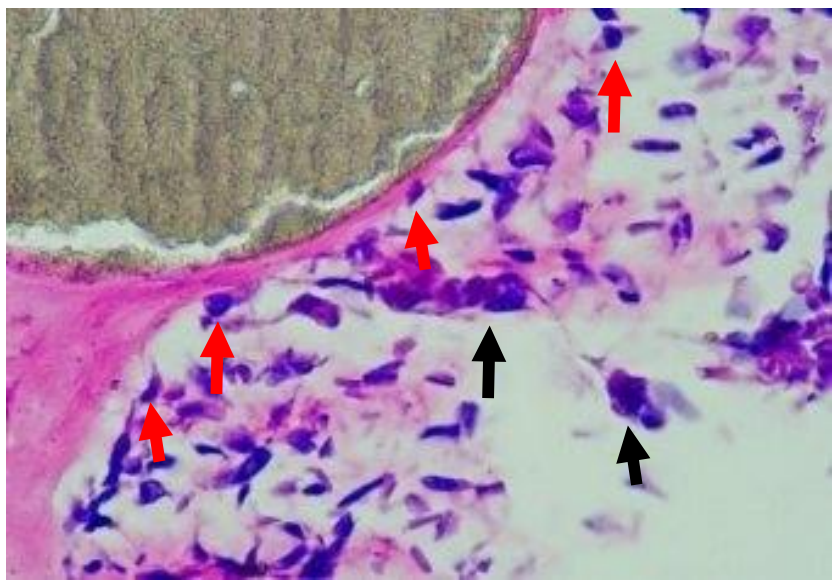

Figure 3. Microscopic image of osteoblast and osteoclast observation week II in the treatment group (DFDBBX).

The osteoblasts in the pictures above are mature osteoblasts; for osteoblasts, the blue color is cuboidal on the side below the graft implantation on the defect with the formation of new bone deposits. As for the flattened osteoclasts with a blue core. Osteoblast cells in the treatment group, positive control and negative control observation week II are indicated by red arrows and osteoclasts are indicated by black arrows.

The results of microscopic observations of osteoblasts and osteoclasts in the treatment group (DFDBBX), positive control group (BBHAX) and negative control group (without graft) with $\mathrm{HE}$ staining at the time of observation week IV can be observed in the image below:

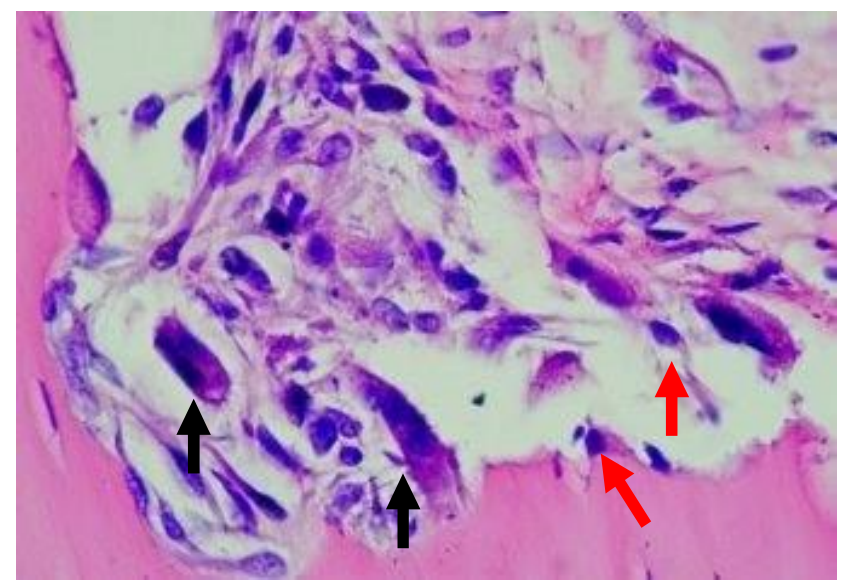

Figure 4. Microscopic images of osteoblasts and osteoclasts observed at week IV in the negative control group (without graft implantation) 


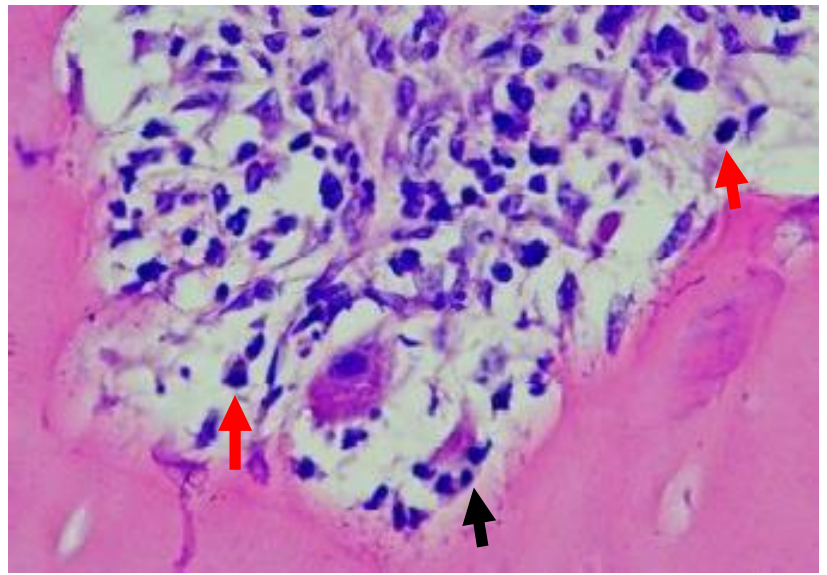

Figure 5. Microscopic images of osteoblasts and osteoclasts observed week IV in the positive control group (BBHAX)

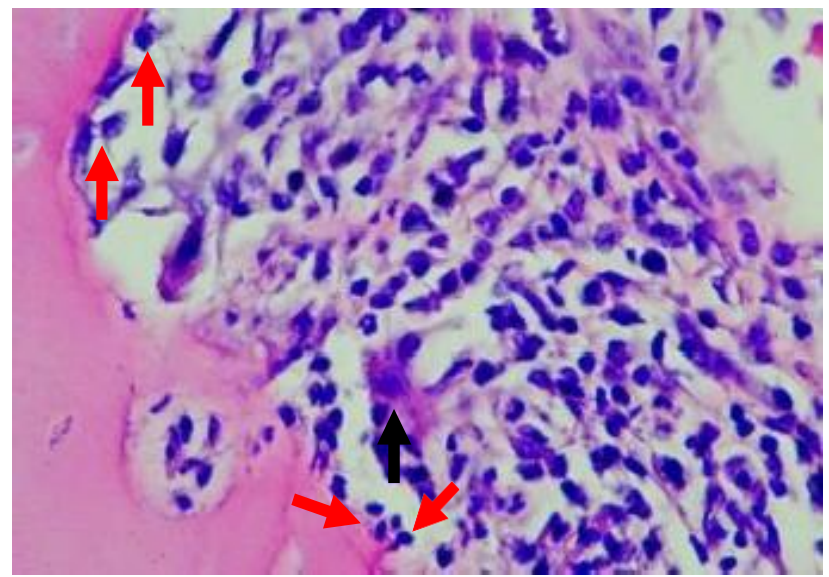

Figure 6. Microscopic images of osteoblasts and osteoclasts observed week IV in the treatment control group (DFDBBX)

The osteoblast in the picture above is a picture of mature osteoblast cells that are cuboidal in shape, dark blue in color, on the underside of the graft on the defect, while the osteoclast cells are flat with a nucleus. Osteoblast cells at week IV are indicated by red arrows and osteoclast cells by black arrows. The impression of the picture on the fourth week of observation, the osteoblast cells in the treatment group looked paler in color, flatter and more amorphous in shape and thinned with irregular edges.

The results of the expression of type 1 collagen were obtained from the results of immunohistochemical examination. The results of microscopic observations of type 1 collagen expression in the negative control group (without graft), positive control (BBHAX) and treatment (DFDBBX) during the second week of observation can be observed in the image below. 


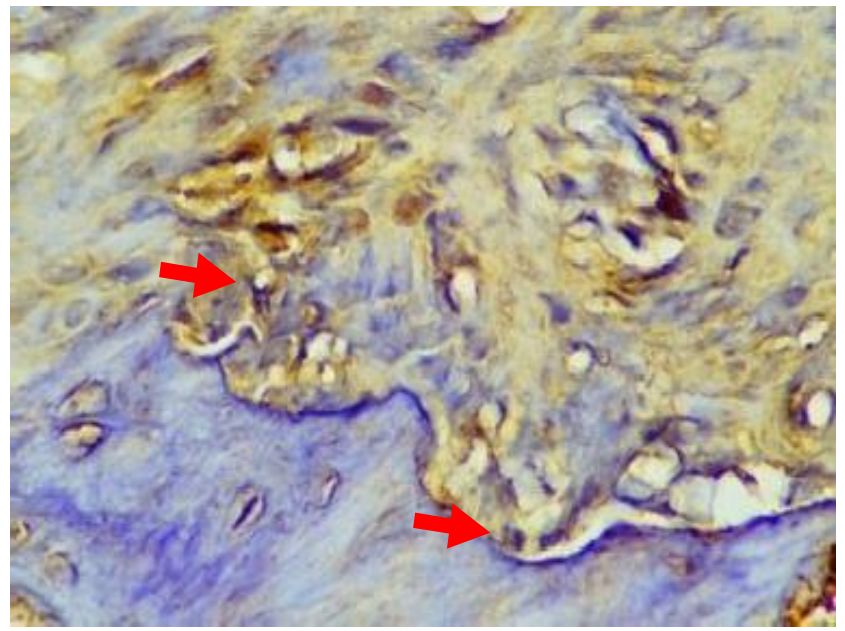

Figure 7. Microscopic appearance of collagen type 1 expression on week II observations in the negative control group (without graft implantation).

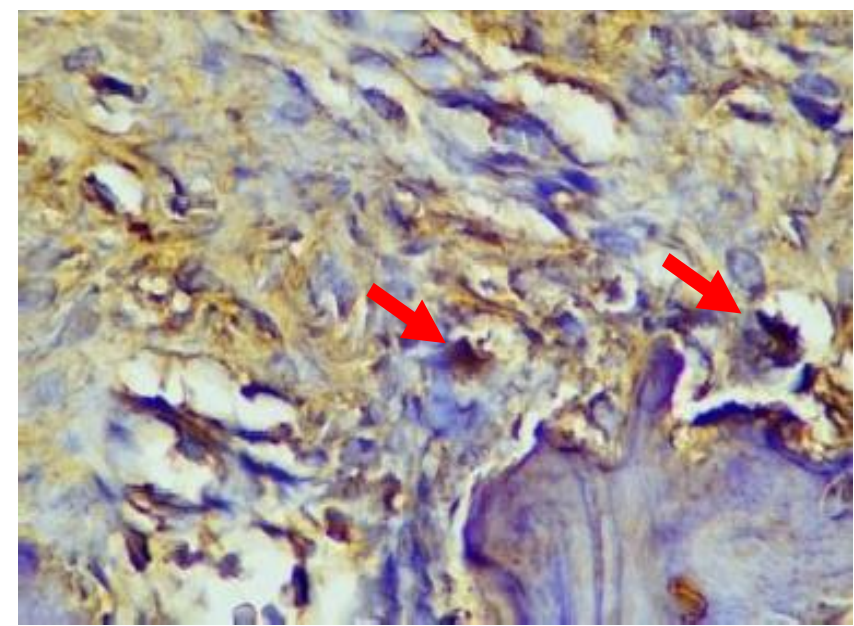

Figure 8. Microscopic appearance of collagen type 1 expression on week II observation in the positive control group (BBHAX).



Figure 9. Microscopic description of the expression of collagen type 1 observation week II in the treatment group (DFDBBX) 
The picture above shows a description of the expression of collagen 1 from the negative control group, positive control and treatment group shown by red arrows. Type 1 collagen is expressed by mature osteoblasts. It is clearly visible at the edges of the post-graft implantation defect. Type 1 collagen is an early product of new bone formation. The impression of brown luminescence produced by osteoblasts in the positive control group compared to the negative control group was almost the same in number.

The results of microscopic observations of type 1 collagen expression in the negative control group (without graft), positive control (BBHAX) and treatment (DFDBBX) during the fourth week of observation can be observed in the image below.

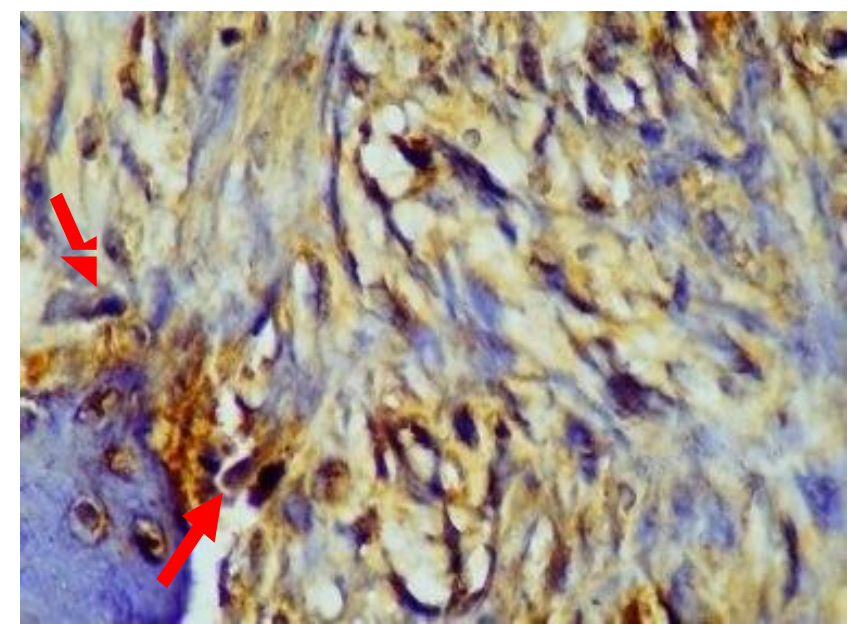

Figure 10. Microscopic appearance of collagen type 1 expression in week IV observation in the negative control group (without graft implantation).

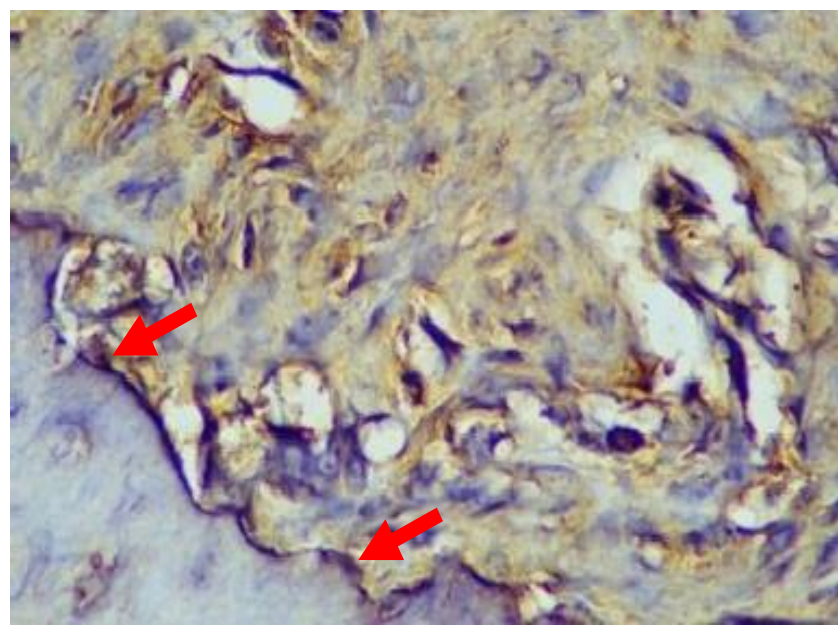

Figure 11. Microscopic description of collagen type 1 expression during week IV observation in the positive control group (BBHAX). 


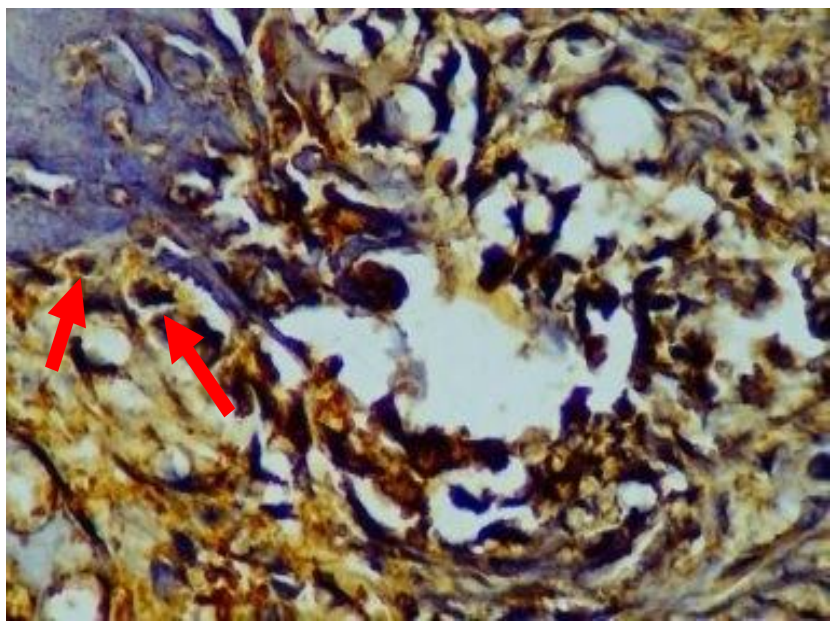

Figure 12. Microscopic description of the expression of collagen type 1 observation week IV in the treatment group (DFDBBX).

In the picture above, a microscopic picture of the expression of type 1 collagen is shown by the red arrow. Expression of type 1 collagen is expressed by mature osteoblasts. In the treatment group, the observation week IV showed that the expression of type 1 collagen was getting clearer and its luminescence was getting clearer when compared to the positive and negative control groups.
The results of microscopic observations of alkaline phosphatase expression in the negative control group (without graft), positive control (BBHAX) and treatment (DFDBBX) during the second week of observation can be observed in the image below.

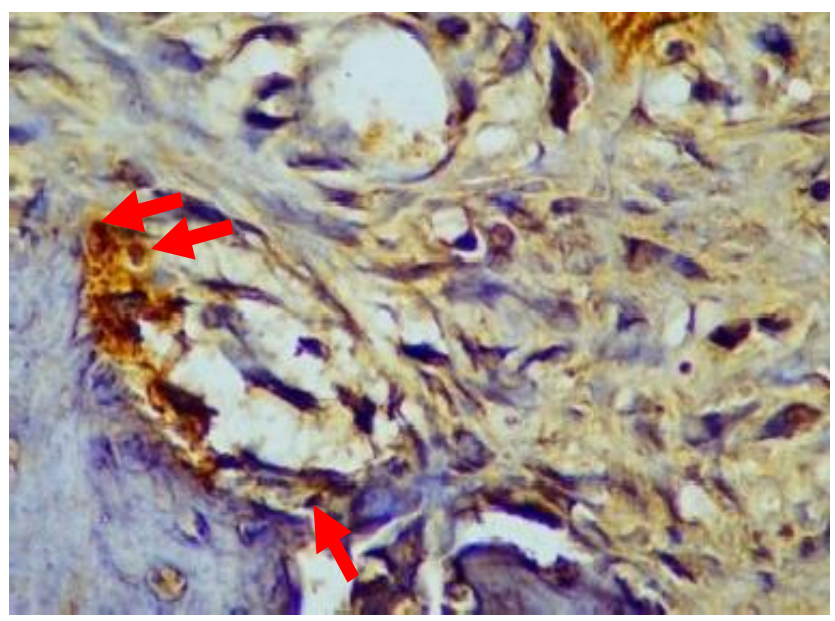

Figure 13. Microscopic view of alkaline phosphatase expression observed in week II negative control group (without graft implantation) 




Figure 14. Microscopic picture of alkaline phosphatase expression observation week II positive control group (BBHAX)

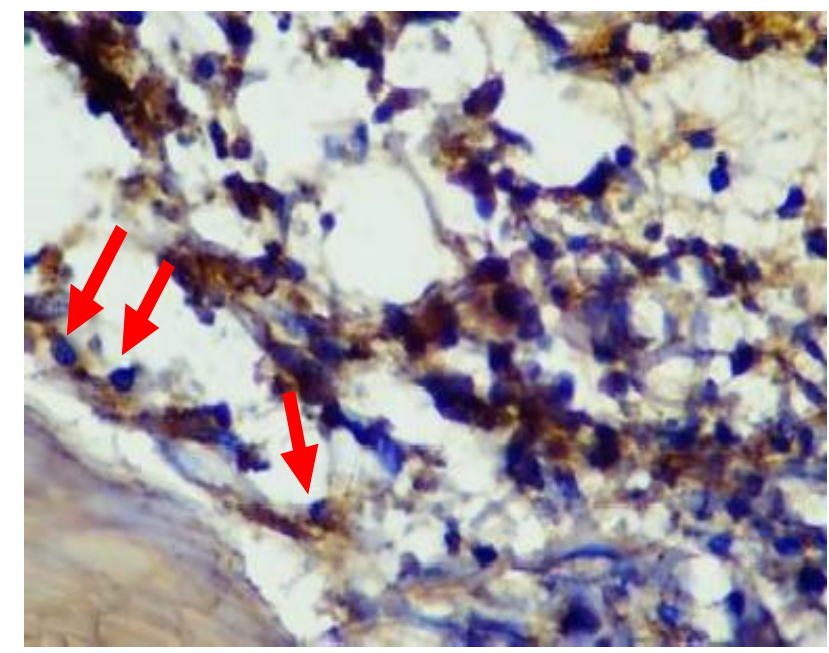

Figure 15. Microscopic picture of alkaline phosphatase expression observed in week II treatment group (DFDBBX)

Alkaline phosphatase is an expression of the expression of alkaline phosphatase is a osteoblast cells which is used as a marker of the brown glow which is indicated by a red arrow phosphorescence process due to the reaction of in the figure.

alkaline phosphatase antibodies. The result of




Figure 16. Microscopic view of alkaline phosphatase expression during week IV of negative control group (without graft implantation)

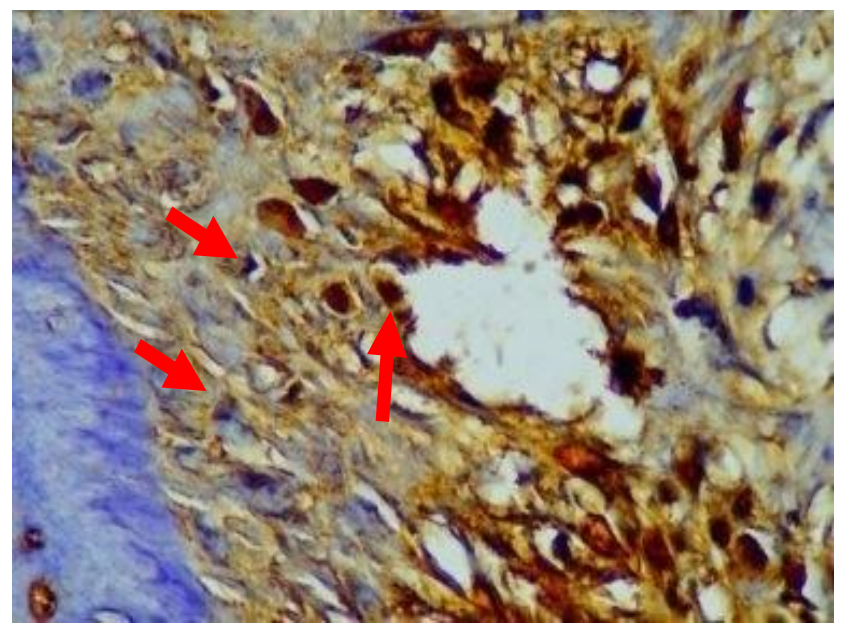

Figure 17. Microscopic picture of alkaline phosphatase expression observation week IV positive control group (BBHAX)

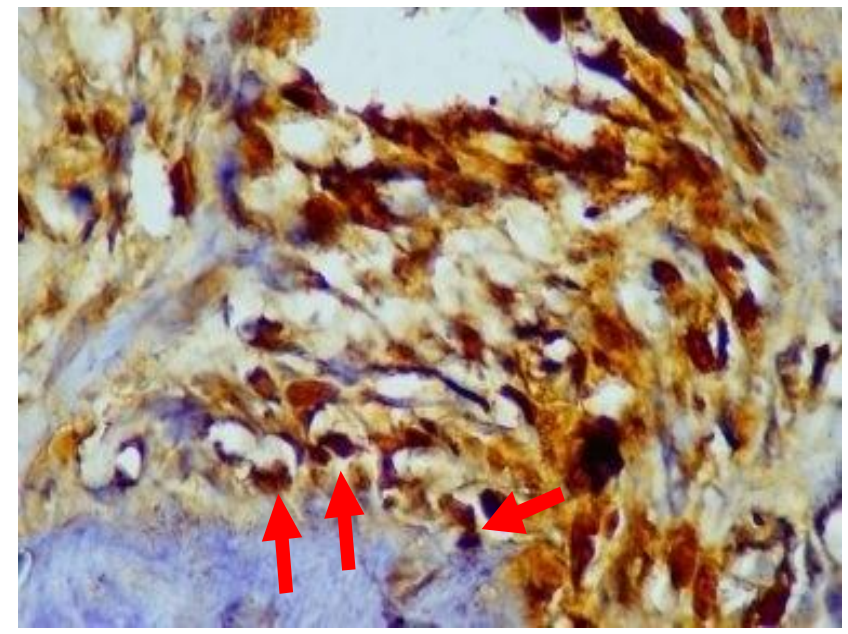

Figure 18. Microscopic description of alkaline phosphatase expression observation week IV treatment group (DFDBBX)

The results of the fourth week of observation, the impression seen in the treatment group of osteoblast cells that glowed brown was almost the same as compared to the positive control group. The visual impression forms bundles and webbing that resembles woven fiber. In the picture in the negative control group the luminescence expression of alkaline phosphatase is weaker.

Description of post-treatment osteoblasts negative control (without graft), positive control (BBHAX), and treatment (DFDBBX) is contained in the table below. 
Table 1. Descriptive statistics of osteoblasts

\begin{tabular}{|c|c|c|c|c|c|c|}
\hline \multirow{2}{*}{$\begin{array}{c}\text { Observation } \\
\text { time }\end{array}$} & \multicolumn{2}{|c|}{ Negative Control } & \multicolumn{2}{c|}{ Positive Control } & \multicolumn{2}{c|}{ Treatment } \\
\cline { 2 - 7 } Averag & SD & $\begin{array}{c}\text { Averag } \\
\mathbf{e}\end{array}$ & SD & $\begin{array}{c}\text { Averag } \\
\mathbf{e}\end{array}$ & SD \\
\hline Week II & 6,3 & 1,0665 & 8.05 & 2.0417 & 12.45 & 2.9706 \\
\hline Week IV & 9 & 2,5799 & 10,14 & 4,2152 & 15,15 & 2,77037 \\
\hline
\end{tabular}

Figure 19. Diagram of mean osteoblasts in each group DFDBBX, BBHAX, and negative control

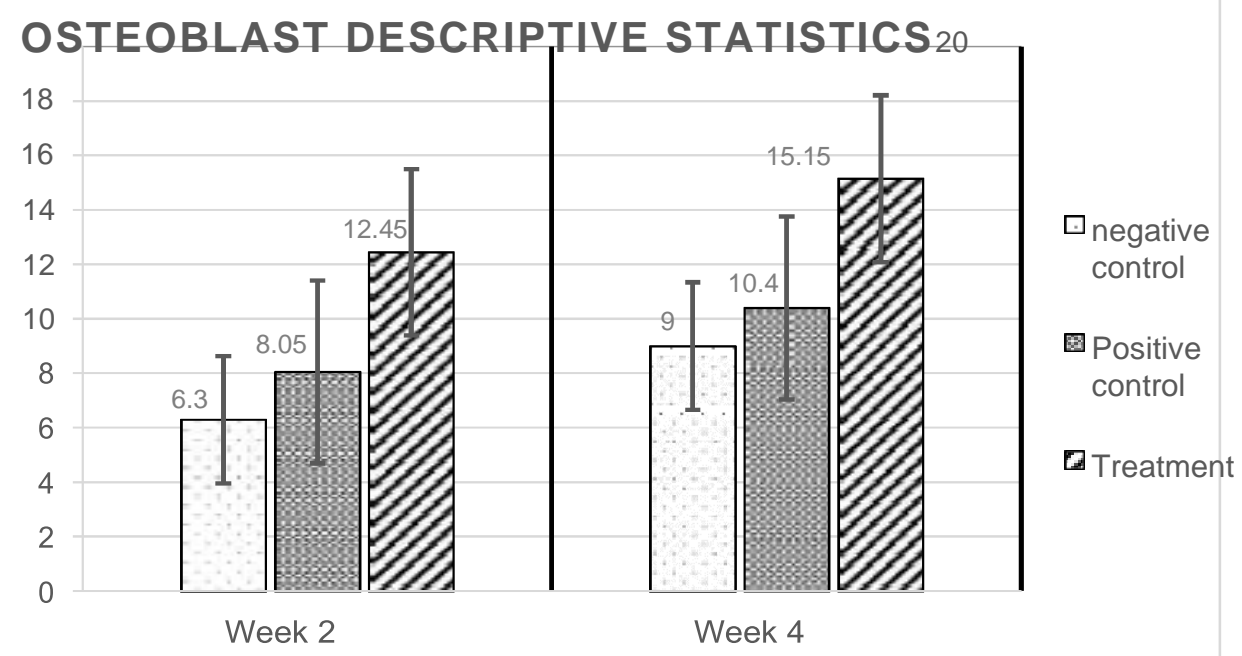

The results of the observations are in the homogeneity tests and different variance tests. form of quantitative data and then statistical The data are differentiated respectively based on analysis is carried out with several parameter the variables and time of observation. The results tests, which include distribution tests, of the analysis can be seen in the table below.

Table 2. Normality test (saphiro Wilk) of osteoblast . research data

\begin{tabular}{|c|c|c|c|c|c|c|}
\hline $\begin{array}{c}\text { Observation } \\
\text { time }\end{array}$ & \multicolumn{2}{|c|}{$\begin{array}{c}\text { Negative } \\
\text { control } \\
\text { (without graft) }\end{array}$} & \multicolumn{2}{|c|}{$\begin{array}{c}\text { positive } \\
\text { control } \\
\text { (BBHA) }\end{array}$} & \multicolumn{2}{|c|}{$\begin{array}{c}\text { Treatment } \\
\text { (DFDBBX) }\end{array}$} \\
\cline { 2 - 7 } & Sig. & Conclusion & Sig. & $\begin{array}{c}\text { Conclusio } \\
\text { n }\end{array}$ & Sig. & $\begin{array}{c}\text { Conclusi } \\
\text { on }\end{array}$ \\
\hline Week II & 0,150 & Normal & 0,468 & Normal & 0,459 & Normal \\
\hline Week IV & 0,817 & Normal & 0,637 & Normal & 0,568 & Normal \\
\hline
\end{tabular}

The results of the osteoblast difference test (ANOVA) at week II and IV observations between the negative control, positive control (BBHAX) and treatment (DFDBBX) groups showed that there was a significant difference $(\mathrm{p}<0.05)$ between the three groups (table 5.4). Then a different test was carried out between each treatment group with the Tukey post hoc difference test 
Table 3. Test of differences between observed osteoblasts and ANOVA

\begin{tabular}{|l|l|l|l|}
\hline Observation time & Different test & $\boldsymbol{p}$-value & Conclusion \\
\hline Week II & Between groups & 0,002 & Significant \\
\hline Week IV & Between groups & 0,029 & Significant \\
\hline
\end{tabular}

The next test is the difference test between osteoblast variables. This test is to see the difference between the negative control group, positive control and treatment week II compared to week IV. The data that appears is parametric data, so the test used is an unpaired difference test, the test results are shown in the table.

Table 4. Different test of osteoblast group variables with unpaired difference test

\begin{tabular}{|c|c|c|}
\hline Treatments Pair & $\boldsymbol{p}$-value & Conclusion \\
\hline $\begin{array}{c}\text { Treatments Pair } \\
\text { Negative Control (without graft) week II vs } \\
\text { IV }\end{array}$ & 0,63 & Not significant \\
\hline Positive Control (BBHAX) week II vs IV & 0,288 & Not significant \\
\hline Treatment (DFDBBX) week II vs IV & 0,176 & Not significant \\
\hline
\end{tabular}

\section{DISCUSSION}

This study is a true experimental study on experimental animals with a post test only control group design on the mandible of experimental animals in the form of New Zealand White Rabbit rabbits. The purpose of this study was to determine the differences in the bone healing process of the implantation of the DFDBBX bone graft in mandibular bone defects compared to the BBHAX bone graft. The sample was divided into 3 groups, each group consisting of 5 experimental animals.

Manufacture of defects in the rabbit mandible using a low speed tripine bur accompanied by irrigation of $0.9 \% \mathrm{NaCl}$ solution to avoid the heat effect caused by the rotation of the bur eye on the bone around the defect. In bone defects there will be an endochondral ossification process that is mechanically unstable, then cartilage tissue will form cartilaginous callus in the form of soft callus occurring on days 7-9 after the bone defect occurs. A number of growth factors transforming growth factors (TGF- $\beta$ ), bone morphogenetic proteins (BMPs), plateletderived growth factors (PDGF), fibroblast growth factors (FGF) and insulin-like growth factors (IGFs) simultaneously contribute to the occurrence of chondro-osteogenesis. . The process of repairing damaged bones requires an adequate blood supply, revascularization is important for successful bone repair. The next process of bone regeneration will occur in the primary soft cartilage callus resorption and then replaced with hard bone callus (hard bony callus).

The next calcification process will occur with the role of mitochondria which accumulate 
calcium granules, then transported to the extracellular matrix where precipitation occurs with phosphate and mineral deposits and apatite crystals are formed. The peak of hard callus formation in animal studies was day 14 . The hard callus formation is then replaced by cartilage that has undergone calcification with woven bone, mechanically the callus will be more structured and stiff. In the remodeling phase, a second resorption phase occurs, where the hard callus will be replaced into lamellar bone with the medullary cavity in the central part. The remodeling process occurs resorption of hard callus by osteoclasts and deposition of lamellar bone by osteoblasts in a balanced manner, starting from the 3 rd to 4 th week and resulting in complete regeneration of bone structure can occur in several years. In the remodeling phase, the process of bone formation occurs according to its origin (resharping). This process is important for maintaining bone integrity and there must be a balance between bone deposition by osteoblasts and bone resorption by osteoclasts (Aql et al., 2008; Marx, 2007).

Immunohistochemistry (IHC) is a method for demonstrating the distribution and location of proteins in tissue sections. Although less quantitatively sensitive than immunoassays such as western blotting or ELISA, the IHC assay allows observation of intact tissue. Antibodyantigen interactions are then visualized using chromogenic detection, in which the enzyme conjugated to the antibody cleaves the substrate to produce a colored precipitate at the protein site, or fluorescent detection, in which the fluorophore is conjugated to the antibody and can be visualized using a fluorescence microscope (Knabe et al., 2005).

The application of Demineralized FreezeDried Bone Xenograft bone graft material in experimental animals can be based on the research conducted by (Ferdiansyah, Utomo, Suroto, \& Gaol, 2017) regarding the immune reaction of DFDBBX bone graft material to tissue, showing no significant increase in immunoglobulin $\mathrm{G}$ in the first, second and second weeks. fourth. Research by (Kamadjaja et al., 2017), showed that demineralized freeze-dried bovine cortical bone membrane (DFDBCBM) has optimal potential for application as membrane guided bone regeneration, undergoes gradual biodegradation and has biocompatibility that can be accepted by body tissues.

The results of statistical analysis on the osteoblast count showed an increase in the treatment group (DFDBBX bone graft) compared to the negative control group (without bonegraft application) both at week II to week IV. This proves that DFDBBX as a whole has a better potential for osteoinduction than without bone grafting on bone defects.

Meanwhile, the observation of the number of osteoblasts in the treatment group compared to the positive control group (BBHAX bone graft) showed a significant difference in week II. However, at week 4, the number of osteoblasts did not appear different. This study is in line with research conducted by (Ferdiansyah, Utomo, Suroto, Martanto, et al., 2017) which showed that there was the highest increase in the number of 
osteoblast cells at week II in the implantation of demineralized dried cortical bone grafts for femoral bone defects in white rabbits. In the fracture healing process, TGF- $\beta$ plays a role in initiating repair, chondrogenesis and bone formation. TGF- stimulates gene expression for callus formation, stimulates mesenchymal cell proliferation and differentiation of osteoblasts and chondrocytes (Buckwalter, 2008; Wiss Ronal, 2011).

Observations on week II, the number of osteoclasts in the treatment group compared to the positive control group (BBHAX) did not differ, while the treatment group (DFDBBX) and the positive control group (BBHAX) compared to the negative control group showed significant differences. Week IV observations showed a significant difference between the treatment group and the positive control group and the negative control group.

In the observation, the mean number of osteoclasts in the negative control group (without graft implantation) based on the time of observation showed no difference in both the II and IV weeks, but on the descriptive graph it tended to increase compared to the pattern in the

\section{REFERENCES}

Aql, Z., Alagl, A., Graves, D., Gerstenfield, L., \& Einhorn, T. (2008). Molecular Mechanisms Controlling Bone Formation During Fracture Healing and Distraction Osteogenesis. Journal Dent Res, 87(2), 108-118. positive and treatment groups which tended to decrease. Observation of the amount of alkaline phosphatase expression in week II there was a difference between DFDBBX and BBHAX. (Birmingham et al., 2012) stated that the process of osteogenic differentiation in MSCs in vitro caused an increase in ALP levels until day 14 which was marked by ALP protein expression and would decrease after 14 days and above, which was followed by an increase in osteocalcin and osteopontin expression and resulted in calcium deposition. and phosphate. Meanwhile, at week IV between DFDBBX and BBHAX there was no significant difference. This can occur because the osteoinduction process in the two bone grafts is different, where DFDBBX is induced by BMP-2, namely the MAPK cascade or through Smad1/5/8 (Xu et.al., 2015) first while BBHAX stimulates in vitro Mesenchymal stem cell differentiation directly without going through the BMP pathway, namely through free ions of Calcium and Phosphate which changes the atmosphere of the medium (environment) (Barradas et al., 2011) which will activate the Notch signaling pathway.

Barradas, A., Yuan, H., Blitterswijk, C. A. V, \& Habibovie, P. (2011). Osteoinductive biomaterials: current knowledge of properties, experimental models and biological mechanisms. European Cells and Materials, 21 2012, 407-429.

Birmingham, E., Niebul, G., McHugh, P., Shaw, G., Barry, F., \& McNamara, L. (2012). 
Osteogenic differentiation of mesenchymal stem cells is regulated by osteocyte and osteoblast cells in a simplified bone niche. European Cells and Materials, 23 2012, $13-27$.

Buckwalter, J. (2008). Orthopaedic Basic Science Biology and Biomechanics of the Musculoskletal System. American Academy of Orhopaedic Surgeon 2nd Edition, 372395.

Buser, D. (2009). 20 Years of Guided Bone Regeneration in Implant Dentistry 2nd ed. Quintessence Publishing Company.

Cohen, R., Mullarky, R., Noble, B., Comeau, R., \& Neiders, M. (1994). Phenotypic characterisation of mononuclear cells following anorganic bovine bone implantation in rats. Journal Periodontal, 65, 1008-1015.

Farzad, M., \& Mohammadi. (2012). Guided bone regeneration: a literature review. Johoe 1,1 , 3-18.

Ferdiansyah. (2007). Use of freeze-dried Iiradiated Bones in Orthopaedic Surgery. In Radiation in Tissue Banking Basic Science and Clinical Applications of Radiated Tissue Allograft. World Scientific Singapore, 317-326.

Ferdiansyah, M., Utomo, D. N., Suroto, H., Martanto, T. W., Edward, M., \& Gaol, I. L. (2017). Comparative Effectiveness of Bone Grafting Using Xenograft Freeze-Dried Cortical Bovine, Allograft Freeze-Dried Cortical New Zealand White Rabbit, Xenograft Hydroxyapatite Bovine, and
Xenograft Demineralized Bone Matrix Bovine in Bone Defect of Femoral Di. International Journal of Biomaterials, 2017.

Ferdiansyah, Utomo, D. N., Suroto, H., \& Gaol, I. L. (2017). Immunogenicity of Bone Graft Using Xenograft Freeze-Dried Cortical Bovine, Allograft Freeze-Dried Cortical New Zealand White Rabbit, Xenograft Hydroxyapatite Bovine, And Xenograft Demineralized Bone Matrix Bovine In Bone Defect Of Femoral Diaphysis White. The Veterinary Medicine International Conference KnE Life Sciences, 344-355.

Hallman, M., \& Thor, A. (2008). Bone Substitutes and Growth Factors as an Alternative/Complement to Autogenous Bone for Grafting in Implant Dentistry. Journal Compilation Periodontology, 47, 172-192.

Hernigou, Poignard, A., Beaujean, \& Rouard. (2005). Percutaneous autologous Bone marrow grafting for nonunions; influence of the number and concentration of progenitor cells. J Bone Joint Surg, 86, 1430-1437.

Hislop, W. S., Finlay, P. M., \& Moos, K. P. (1993). A preliminary study into the use of anorganic bone in oral and maxillofacial surgery. Br. J. Oral Maxillofac. Surg, 31, $149-153$.

Kamadjaja, D. B., Harijadi, A., Soesilowati, P., Eny, W., Nurul, M., Akhsanal, F., Fika, R. A., Roberto, S., Soesanto, Djodi, A., Andra, R., Peter, A., \& Coen, P. (2017). Demineralized Freeze-Dried Bovine 
Cortical Bone: Its Potential for Guided Bone Regeneration Membran. International Journal of Dentistry, 1-9.

Knabe, C., Kraska, B., Koch, C., Gross, U., Zreiqat, H., \& Stiller, M. (2005). A method for immunohistochemical detection of osteogenic markers in undecalcified bone sections. Biotechnic \& Histochemistry, 81(1), 31-39.

Marx, R. (2007). Bone and Bone Grafting
Healing. Oral Maxillofacial Surg Clin N Am, 19, 455-466.

Stephan, E. B., Jang, D., Lynch, S., Bush, P., \& Dziak, R. (1999). Anorganic bovine bone supports osteoblastic cell attachment and proliferation. Journal Periodontal, 70, 364369.

Wiss Ronal, A. (2011). Fracture master techniques in Orthopaedic surgery 3th edition. Lippicott William \& Wilkins. 